\title{
Effectiveness Various Physical Exercise to Decrease Glycemic Control in Patient with Diabetes Mellitus : A Systematic Review
}

\author{
Tifanny Gita Sesaria, Kusnanto*, Abu Bakar \\ Nursing Faculty, Ailangga University, Indonesia \\ *kusnanto@fkp.unair.ac.id
}

\begin{abstract}
Diabetes mellitus is a major global health problem that affect almost 400 million people in the world. Increasing prevalence of T2DM diabetes is followed by some causal factor such as obesity, age and sedentary lifestyle. Physical exercise have been considered as on of 'gold standart intervention' in management of type 2 diabetes. Types of exercise and physical activity that related with $\mathrm{T} 2 \mathrm{DM}$ such as aerobic exercise, also resistance (strength) training includes elastic resistance bands. Nevertheless, there is still a low progress and prevalence of exercise in diabetes population.

The aim of our study is to present the effect of various physical exercise such aerobic, resistance exercise also combined aerobic and resistance exercise to decrease Hba1c and blood glucose level.

We search the scientific literature about diabetes and physical exercise in the database such as Scopus, PubMed, ProQuest, Spinger Link and Science Direct for original and full research and then make a systematic review from 1300 articles retrieved, 18 studies include final criteria and obtained using PRISMA (Preferred Reporting Items for Systematic Reviews and Meta-Analyses) method.

From the study review combined aerobic physical exercise and resistance exercise improved the glycemic control of T2DM and it was widely used and significant for glycemic control.

This systematic review could be used as evidence when increase combine aerobic and resistance exercise as choice physical exercise interventions for the purpose of glycemic control.
\end{abstract}

Keywords: Aerobic, Diabetes, HbA1c, Physical Exercise, Resistance Exercise 


\section{STRADA Jurnal Ilmiah Kesehatan}

DOI: $10.30994 /$ sjik.v9i2.318

ISSN: 2252-3847 (print); 2614-350X (online)

Vol.9 No.2 November 2020 Page.456-470

\section{BACKGROUND}

Diabetes mellitus is a major global health problem that affect almost 300 million people in the world (Yubo Liu \& Ye, 2019) According to the latest statistics from the International Diabetes Association, the incidence of diabetes in adults (20-79 years) has risen to 425 million worldwide, and this number is projected to increase to 629 million by 2045. Currently, the largest number of people with diabetes (20-79 years) are in China (114 million), India (73 million), and in the USA (30 million) (IDF, 2017). Increasing prevalence of T2DM diabetes is followed by some causal factor such as obesity, age and sedentary lifestyle. Physical exercise have been considered as on of 'gold standart intervention' in treatment of type 2 diabetes. Types of exercise and physical activity that suitable with T2DM such as aerobic exercise, activities such as walking, cycling, jogging, and swimming also resistance (strength) training includes elastic resistance bands (Colberg \& Sigal, 2016). Nevertheless, there is still a low progress and prevalence of exercise in diabetes population.

Physical activity, especially aerobic exercise (AE) has consistently been reported to decrease the glycemic control, insulin resistance and dyslipidemia in patients with T2DM (Dixit \& Maiya, 2017).

Another recommendation of $\mathrm{AE}$, recent findings have a new intervention about the resistance exercise (RE) in efficient management of diabetes. Recommended incorporation from The American College of Sports Medicine (ACSM) about progressive RE to treat T2D (Chodzko-Zajko \& Proctor, 2019). The American Diabetes Association (ADA) claimed that both intervention like resistance exercise and aerobic training can improve insulin action, and assist in management of blood glucose, lipids, cardiovascular risk factors, and HbA1c (Colberg \& Sigal, 2016). However, recenlty RCT reported that 52week RE without AE significantly lowers the HbA1c in patients with T2DM (Najafipour \& Mobasseri, 2017). A systematic review indicated that both RE and AE, combine RE and $\mathrm{AE}$ are effective in manage diabetes glycemic control (decreased glycosylated hemoglobin (HbA1c)), risk factors or safety about newly RE is must be concern in many condition of T2DM patients (Siavoshy \& Heidarianpour, 2017) (AminiLari \& Fararouei, 2017). Increasing curiousity, functionality and advantages of RE in recent studies could be following with explore further details on the influence of RE, RE variables, RE intensity and how far it can responsible for beneficial effects in controlling T2DM.

On the other hand, aerobic exercise (AE) as a conventional physical exercise for T2DM including jogging, brisk walking, cycling, and swimming recruits a large group of muscles to perform, and usually requires prolonged time to manage T2DM (Ghalavand \& Rooholah, 2016) (Gholami \& Nikookheslat, 2018). Additionally, no meta-analysis has compared the effects of different $\mathrm{AE}, \mathrm{RE}$ combine with $\mathrm{AE}$ also RE intensities on glycemic control in patients with T2DM.The data collected from all available sources were included into systematic review and examined effective intensity of RE in controlling the $\mathrm{HbA} 1 \mathrm{c}$ and blood glucoselevel in patients with T2DM. The aim of the present study was to review the findings from the randomized scientific published literature studies focusing on the T2DM patients used various types of exercise and their effect on glycemic control. 


\section{STRADA Jurnal Ilmiah Kesehatan}

DOI: $10.30994 /$ sjik.v9i2.318

ISSN: 2252-3847 (print); 2614-350X (online)

Vol.9 No.2 November 2020 Page.456-470

\section{METHODS}

\section{Inclusion and Exclusion Criteria}

The inclusion and exclusion criteria used for this study have been presented in Table 1 .

Table 1. Inclusion and exclusion criteria

\begin{tabular}{lll}
\hline & \multicolumn{1}{c}{ Inclusion criteria } & \multicolumn{1}{c}{ Exclusion criteria } \\
\hline 1. & $\begin{array}{l}\text { The study design specifically evaluated the } \\
\text { effect of physical exercise on glycemic } \\
\text { control such as HbA1c and glucose level }\end{array}$ & $\begin{array}{l}\text { Studies that evaluate the effect of physical exercise } \\
\text { on other chronic diseases }\end{array}$ \\
\hline 2. & This study focused on T2DM patients & $\begin{array}{l}\text { Studies that evaluate the effect of physical exercise } \\
\text { on children with diaberes or mothers with } \\
\text { gestational diabetes }\end{array}$ \\
\hline 3. & Studies that were published in English & Patient with complications or another disease \\
\hline T. & $\begin{array}{l}\text { The study had one of the following study } \\
\text { designs: randomized controlled trial and } \\
\text { a before-after trial }\end{array}$ & \\
\hline
\end{tabular}

\section{Seacrhing strategy}

This study is about systematic review on the effectiveness of various physical exercise for glycemic control for T2DM patients. A comprehensive literature search was conducted using Scopus, PubMed, ProQuest, Spinger Link and Science Direct journal databases. This study began with determining the topic, searching for original articles and matched with the inclusion and exclusion criteria. The keywords that used are aerobic, diabetes, Hbalc, physical exercise, resistance exercise. Article selection was done according to the PRISMA guidelines.The comprehensive steps of the selection process and number of articles in each step were presented as a flow diagram in Figure 1.

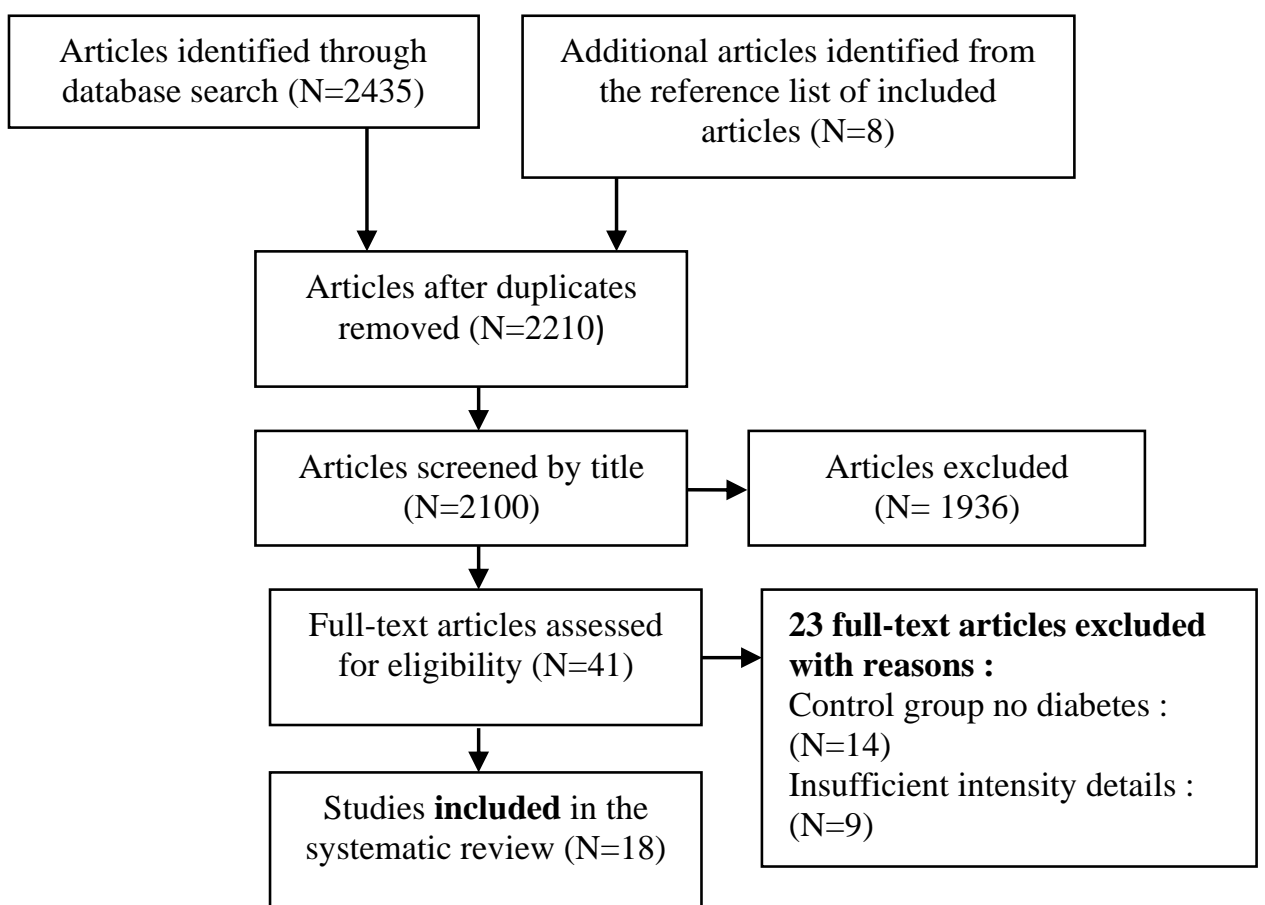

Figure 1. Reporting Items for Systematic Review and Meta-analysis (PRISMA) flow diagram of study selection. 


\section{STRADA Jurnal Ilmiah Kesehatan}

DOI: $10.30994 /$ sjik.v9i2.318

ISSN: 2252-3847 (print); 2614-350X (online)

Vol.9 No.2 November 2020 Page.456-470

\section{Data Extraction and quality assesment}

The title, abstract and the article were reviewed independently according to inclusion and exclusion criteria. The information that should be considered each study that the full text study consist of authors, date and year of study, population, exercise type, outcome measures, study design, duration of the intervention and its intensity. The quality assessment for each selected study was conducted using the PRISMA method and risk of bias assesment study was conducted using Joanna Briggs Institute (JBI) checklist.

\section{Descriptions of the included articles}

This systematic review of 18 study, total 985 patients with T2D were enrolled from smallest particpants are 12 and the largest are 265 participants. The selected studies were including from Iran, Canada, China, Taiwan, Germany, Norway, India, Israel and South Korea. The included trials according to inclusion criteria, were published between 2015 until 2020.

\section{RESULTS}

The interventions that used were aerobic exercise (AE), resistance exercise (RE) and a combination of both exercises (AE and RE). Three studies used an aerobic exercise (AE) only intervention. Six study used a resistance exercise (RE) only intervention. Six studies used a combination of aerobic and resistance exercises. One used a traning program and one regular exercise. The intensity rate for all of physical exercise ranged from 2 to 3 times a week for 30 to 60 minutes. All of the study intervention primary outcome for reducing HbA1c and blood glucose level. Samples for the pre and post examination of blood sugar and HbAlc were obtained both before exercise and after the administration of the intervention in intervention and control group.

Time for re-examination of the blood glucose levels and HbAlc are varied from 2 weeks and 3 week. All results of the study stated that combined aerobic and resistance studies gave significant results for glycemic control, blood sugar levels and HbAlc. Combined Aerobic exercise (AE) and Resistance exercise (RE) was the most widely used and modest exercise used to maintain glycemic control. The main characteristics of the included studies have been summarized in Table 2 .

Table 2. Characteristicsof the trials included in the systematic review.

\begin{tabular}{|c|c|c|c|c|c|c|c|}
\hline No & Title & $\begin{array}{l}\text { Year, } \\
\text { Contry }\end{array}$ & $\begin{array}{l}\text { Sample } \\
\text { Size }\end{array}$ & $\begin{array}{l}\text { Type of } \\
\text { Exercise }\end{array}$ & $\begin{array}{c}\text { Intensity of } \\
\text { Exercise }\end{array}$ & $\begin{array}{l}\text { Finding/ } \\
\text { conclusion }\end{array}$ & Reference \\
\hline 1. & $\begin{array}{l}\text { Effects of sprint } \\
\text { interval or combined } \\
\text { aerobic and resistance } \\
\text { training on myokines } \\
\text { in overweight women } \\
\text { with type } 2 \text { diabetes: } \\
\text { A randomized } \\
\text { controlled trial }\end{array}$ & $\begin{array}{l}2018 \\
\text { Iran }\end{array}$ & $\begin{array}{l}52 \\
\text { participant }\end{array}$ & $\begin{array}{l}\text { Sprint } \\
\text { interval } \\
\text { training } \\
(\mathrm{SIT}) \text { or } \\
\text { combined } \\
\text { aerobic and } \\
\text { resistance } \\
\text { training } \\
(\mathrm{A}+\mathrm{R})\end{array}$ & $\begin{array}{l}\text { Resistance } \\
\text { Training: } \\
10 \text { weeks/3 days } \\
\text { per week } \\
\text { duration2-3 } \\
\text { minutes } \\
\text { Aerobic Training: } \\
10 \text { weeks/ } 3 \text { days } \\
\text { per week duration } \\
\text { min } 15-\text { max30 }\end{array}$ & $\begin{array}{l}\text { There was effect of } \\
\text { either exercise } \\
\text { fasting glucose }(\mathrm{p}= \\
0.03) \text { and } \mathrm{HbA} 1 \mathrm{c}(\mathrm{p}= \\
0.006) \text { improved } \\
\text { significantly and } \\
\text { similarly in both } \\
\text { exercise groups } \\
\text { compared to } \\
\text { controls }\end{array}$ & $\begin{array}{l}\text { (Banitalebi } \\
\text {, Kazemi, } \\
\& \\
\text { Faramarzi, } \\
\text { 2019) }\end{array}$ \\
\hline & & & & & $\begin{array}{l}\text { Sprint interval } \\
\text { training (SIT): }\end{array}$ & & \\
\hline
\end{tabular}


STRADA Jurnal Ilmiah Kesehatan

DOI: $10.30994 /$ sjik.v9i2.318

ISSN: 2252-3847 (print); 2614-350X (online)

Vol.9 No. 2 November 2020 Page.456-470

\begin{tabular}{|c|c|c|c|c|c|c|c|}
\hline No & Title & $\begin{array}{l}\text { Year, } \\
\text { Contry }\end{array}$ & $\begin{array}{c}\text { Sample } \\
\text { Size }\end{array}$ & $\begin{array}{l}\text { Type of } \\
\text { Exercise }\end{array}$ & $\begin{array}{c}\text { Intensity of } \\
\text { Exercise } \\
\end{array}$ & $\begin{array}{c}\text { Finding/ } \\
\text { conclusion }\end{array}$ & Reference \\
\hline & & & & & $\begin{array}{l}10 \text { weeks/ } 3 \text { days } \\
\text { per week } \\
\text { duration } 4 \times 30 \text { s }\end{array}$ & & \\
\hline 2. & $\begin{array}{l}\text { Effects of exercising } \\
\text { before breakfast on } \\
\text { the health of T2DM } \\
\text { patients-A } \\
\text { randomized } \\
\text { controlled trial }\end{array}$ & $\begin{array}{l}2019 \\
\text { Germany }\end{array}$ & $\begin{array}{l}30 \\
\text { participant }\end{array}$ & $\begin{array}{l}\text { Training } \\
\text { program }\end{array}$ & $\begin{array}{l}8 \text { weeks/3-5 days } \\
\text { per weeks }\end{array}$ & $\begin{array}{l}\text { Physical training } \\
\text { significantly } \\
\text { dercrease glycated } \\
\text { hemoglobin (HbA1c) } \\
\text { values (absolute } \\
\text { change: } 0.3 \% \text { ) } \\
\text { decreased } \\
\text { significantly from } \\
\text { pre- to post-training. }\end{array}$ & $\begin{array}{l}\text { (Brinkman } \\
\text { n, Weh- } \\
\text { Gray, \& } \\
\text { Brixius, } \\
\text { 2019) }\end{array}$ \\
\hline 3. & $\begin{array}{l}\text { The effect of circuit } \\
\text { resistance training, } \\
\text { empagliflozin } \\
\text { or“"vegeterranean } \\
\text { diet”on } \\
\text { physical and } \\
\text { metabolic function in } \\
\text { older } \\
\text { subjects with type } 2 \\
\text { diabetes: a study } \\
\text { protocol for a } \\
\text { randomized control } \\
\text { trial (CEV-65 trial) }\end{array}$ & $\begin{array}{l}2019 \\
\text { Israel }\end{array}$ & $\begin{array}{l}120 \\
\text { participant }\end{array}$ & $\begin{array}{l}\text { Circuit } \\
\text { resistance } \\
\text { training }\end{array}$ & $\begin{array}{l}10 \text { weeks CRT } \\
3 \text { home } \\
\text { sessions/week; }\end{array}$ & $\begin{array}{l}\text { Glycemic control } \\
\text { FPG and HbA1c } \\
\text { significant dercrease } \\
(p=0.006)\end{array}$ & $\begin{array}{l}\text { (Buch, } \\
\text { Eldor, \& } \\
\text { Kis, 2019) }\end{array}$ \\
\hline 4. & $\begin{array}{l}\text { Efects of Resistance } \\
\text { Exercise on Glycated } \\
\text { Hemoglobin and } \\
\text { Functional } \\
\text { Performance in Older } \\
\text { Patients with } \\
\text { Comorbid Diabetes } \\
\text { Mellitus and Knee } \\
\text { Osteoarthritis: A } \\
\text { Randomized Trial }\end{array}$ & $\begin{array}{l}2020 \\
\text { Taiwan }\end{array}$ & $\begin{array}{l}70 \\
\text { participant }\end{array}$ & $\begin{array}{l}\text { Dynamic } \\
\text { Resistance } \\
\text { Exercise } \\
\text { Group } \\
\text { Isometric } \\
\text { Resistance } \\
\text { Exercise } \\
\text { Group }\end{array}$ & $\begin{array}{l}\text { 12-week } \\
\text { intervention } \\
\text { involve } 8-15 \\
\text { RM, which must } \\
\text { include 5-10 min } \\
\text { of warm-up and } \\
\text { cool-down }\end{array}$ & $\begin{array}{l}\text { For HbA1c level, the } \\
\text { main e ects were not } \\
\text { statistically } \\
\text { significant over time } \\
\text { within groups } \\
(\mathrm{p}=0.167) \text { or } \\
\text { between groups ( } \mathrm{p}= \\
0.462) \text {. Moreover, no } \\
\text { significant time-by- } \\
\text { group interaction was } \\
\text { noted ( } \mathrm{p}=0.957)\end{array}$ & $\begin{array}{l}\text { (Chen, } \\
\text { Shen, \& } \\
\text { Chen, } \\
\text { 2020) }\end{array}$ \\
\hline 5 . & $\begin{array}{l}\text { Effect of moderate- } \\
\text { intensity aerobic } \\
\text { exercise on } \\
\text { glycosylated } \\
\text { haemoglobin among } \\
\text { elderly patients with } \\
\text { type } 2 \text { diabetes \& } \\
\text { peripheral neuropathy }\end{array}$ & $\begin{array}{l}2017 \\
\text { India }\end{array}$ & $\begin{array}{l}87 \text { patient } \\
\text { with } \\
\text { T2DM }\end{array}$ & $\begin{array}{l}\text { Aerobic } \\
\text { exercise }\end{array}$ & $\begin{array}{l}\text { Eight weeks } \\
\text { intervention } \\
\text { Intensity } \\
3 \text { times/week, } 50 \\
\text { minutes, } 8 \text { week }\end{array}$ & $\begin{array}{l}\text { mean values of study } \\
\text { and } \\
\text { control groups for } \\
\text { HbA1c, post-prandial } \\
\text { blood sugar } \\
\text { (PPBS) and fasting } \\
\text { blood sugar (FBS) at } \\
\text { baseline and } \\
\text { eighth weeks is } \\
\text { presented } \\
\text { that there was a } \\
\text { significant difference } \\
\text { in the mean value } \\
\text { of HbA 1c at baseline } \\
\text { and eighth week } \\
\text { between the two } \\
\text { groups (P<0.001) } \\
\text { The mean value of } \\
\text { glycosylated }\end{array}$ & $\begin{array}{l}\text { (Dixit \& } \\
\text { Maiya, } \\
\text { 2017) }\end{array}$ \\
\hline
\end{tabular}


STRADA Jurnal Ilmiah Kesehatan

DOI: $10.30994 /$ sjik.v9i2.318

ISSN: 2252-3847 (print); 2614-350X (online)

Vol.9 No. 2 November 2020 Page.456-470

\begin{tabular}{|c|c|c|c|c|c|c|c|}
\hline No & Title & $\begin{array}{l}\text { Year, } \\
\text { Contry }\end{array}$ & $\begin{array}{l}\text { Sample } \\
\text { Size }\end{array}$ & $\begin{array}{l}\text { Type of } \\
\text { Exercise }\end{array}$ & $\begin{array}{c}\text { Intensity of } \\
\text { Exercise } \\
\end{array}$ & $\begin{array}{c}\text { Finding/ } \\
\text { conclusion }\end{array}$ & Reference \\
\hline & & & & & & $\begin{array}{l}\text { haemoglobin showed } \\
\text { a mean reduction of } \\
0.79 \text { per cent } \\
\text { in the study group } \\
\text { whereas PPBS and } \\
\text { FBS of the two } \\
\text { groups had no } \\
\text { significant difference }\end{array}$ & \\
\hline 6. & $\begin{array}{l}\text { Morning (Fasting) vs } \\
\text { Afternoon Resistance } \\
\text { Exercise in } \\
\text { Individuals With } \\
\text { Type 1 Diabetes: A } \\
\text { Randomized } \\
\text { Crossover Study }\end{array}$ & $\begin{array}{l}2019 \\
\text { Canada }\end{array}$ & $\begin{array}{l}12 \\
\text { participant }\end{array}$ & $\begin{array}{l}\text { Afternoon } \\
\text { Resistance } \\
\text { Exercise }\end{array}$ & $\begin{array}{l}7 \text { am and } 5 \text { pm } \\
\text { per day until } 10 \\
\text { weeks. } \\
\text { Performed } 40 \\
\text { minutes of RE } \\
\text { (three sets of } \\
\text { eight repetitions, } \\
\text { seven exercises, } \\
\text { at the individual's } \\
\text { predetermined } \\
\text { eight repetition } \\
\text { maximum) }\end{array}$ & $\begin{array}{l}\text { Blood glucose rose } \\
\text { with afternoon } \\
\text { exercise }(8.262 .5 \text { to } \\
7.462 .6 \\
\text { mmol/L;P50.031 for } \\
\text { time-by-treatment } \\
\text { interaction). } \\
\text { Mean blood glucose } \\
\text { level after morning } \\
\text { exercise was } 10.4 \\
63.0 \text { mmol/L } \\
\text { compared with } 7.4 \\
62.6 \text { mmol/L } \\
\text { (P50.019) upon } \\
\text { completion of } \\
\text { afternoon exercise. }\end{array}$ & $\begin{array}{l}\text { (Toghi- } \\
\text { Eshghi \& } \\
\text { Reza, } \\
\text { 2019) }\end{array}$ \\
\hline 7. & $\begin{array}{l}\text { Effects of aerobic } \\
\text { training on FBS, } \\
\text { HbA1C,Fructosamine } \\
\text { and plasma lipid } \\
\text { profile in male with } \\
\text { type } 2 \text { diabetes }\end{array}$ & $\begin{array}{l}2016 \\
\text { Iran }\end{array}$ & $\begin{array}{l}20 \\
\text { participant }\end{array}$ & $\begin{array}{l}\text { Aerobic } \\
\text { Training }\end{array}$ & $\begin{array}{l}8 \text { weeks, one day } \\
\text { per weeks } \\
\text { duration from } \\
\text { first meet : } 10 \\
\text { minutes and final } \\
\text { meet } 30 \text { minutes } \\
\text { duration per } \\
\text { weeks. }\end{array}$ & $\begin{array}{l}\text { Eight weeks of } \\
\text { aerobic training } \\
\text { resulted in a } \\
\text { significant decrease } \\
\text { in fasting blood } \\
\text { glucose ( } \mathrm{P}=0.017) \text {, } \\
\text { HbA1C } \\
(\mathrm{P}=0.045) \text {,Fructosami } \\
\text { ne }(\mathrm{P}=0.012) \text { and } \\
\mathrm{LDL}(\mathrm{P}=0.048) \text { and } \\
\text { a significant } \\
\text { increase in } \\
\text { HDL }(\mathrm{P}=0.012) \text { of } \\
\text { exercise } \\
\text { group .There were no } \\
\text { significantly changes } \\
\text { in all variables of } \\
\text { control } \\
\text { group }(\mathrm{P}>0.05)\end{array}$ & $\begin{array}{l}\text { (Ghalavan } \\
\text { d \& } \\
\text { Rooholah, } \\
2016 \text { ) }\end{array}$ \\
\hline 8. & $\begin{array}{l}\text { Effect of aerobic } \\
\text { training on nerve } \\
\text { conduction in men } \\
\text { with type } 2 \text { diabetes } \\
\text { and peripheral } \\
\text { neuropathy: A } \\
\text { randomized } \\
\text { controlled trial }\end{array}$ & $\begin{array}{l}2018 \\
\text { Iran }\end{array}$ & 31 patient & $\begin{array}{l}\text { Aerobic } \\
\text { training }\end{array}$ & $\begin{array}{l}12 \text { weeks } \\
\text { Then, they } \\
\text { underwent } \\
\text { aerobic exercise } \\
\text { program for three } \\
\text { months (walking, } \\
\text { jogging or } \\
\text { running on } \\
\text { treadmill, three } \\
\text { sessions a week, } \\
50 \text { - } 70 \% \text { of heart } \\
\text { rate reserve. } 20 \text { - }\end{array}$ & $\begin{array}{l}\text { Comparing pre-test } \\
\text { versus post-test } \\
\text { values, fasting } \\
\text { glucose levels in the } \\
\text { exercise group } \\
\text { significantly } \\
\text { decreased from } \\
11.22 \pm 3.8 \mathrm{mmol} / \mathrm{L} \text { to } \\
8.58 \pm 2.7 \mathrm{mmol} / \mathrm{L} \\
\text { representing } 23 \% \\
\text { improvement } \\
\text { following exercise }\end{array}$ & $\begin{array}{l}\text { (Gholami } \\
\& \\
\text { Nikookhes } \\
\text { lat, 2018) }\end{array}$ \\
\hline
\end{tabular}


STRADA Jurnal Ilmiah Kesehatan

DOI: $10.30994 /$ sjik.v9i2.318

ISSN: 2252-3847 (print); 2614-350X (online)

Vol.9 No. 2 November 2020 Page.456-470

\begin{tabular}{|c|c|c|c|c|c|c|c|}
\hline No & Title & $\begin{array}{l}\text { Year, } \\
\text { Contry }\end{array}$ & $\begin{array}{c}\text { Sample } \\
\text { Size } \\
\end{array}$ & $\begin{array}{l}\text { Type of } \\
\text { Exercise }\end{array}$ & $\begin{array}{c}\text { Intensity of } \\
\text { Exercise } \\
\end{array}$ & $\begin{array}{c}\text { Finding/ } \\
\text { conclusion }\end{array}$ & Reference \\
\hline & & & & & $\begin{array}{l}45 \mathrm{~min}) \text {. } \\
\text { They were } \\
\text { required to record } \\
3 \text {-day ( } 2 \\
\text { weekdays and } 1 \\
\text { weekend) }\end{array}$ & $\begin{array}{l}\text { program }(\mathrm{P}=0.020) \text {. } \\
2 \text { hpp glucose level } \\
\text { also decreased } \\
\text { significantly from } \\
15.76 \pm 2.5 \mathrm{mmol} / \mathrm{L} \text { to } \\
14.21 \pm 2.5 \mathrm{mmol} / \mathrm{L} \\
\text { indicating } 9 \% \\
\text { reduction in the mean } \\
\text { value }(\mathrm{P}=0.009) . \text { In } \\
\text { the exercise group, } \\
\text { HbA1c } \\
\text { significantly } \\
\text { decreased from } \\
8.3 \% \pm 1.4 \% \text { at week } \\
(0) \text { to } 7.7 \% \pm 1.5 \% \text { at } \\
\text { week }(12) \text { and } \\
\text { statistical } \\
\text { significance } \\
\text { was reached for the } \\
\text { timexgroup } \\
\text { interaction (F= } 7.097 \text {, } \\
\mathrm{P}=0.014) \text {. }\end{array}$ & \\
\hline 9. & $\begin{array}{l}\text { The impact on } \\
\text { glycemic control } \\
\text { through } \\
\text { progressive resistance } \\
\text { training with } \\
\text { bioDensity } \\
\text { in Chinese elderly } \\
\text { patients with type } 2 \\
\text { diabetes } \\
\text { The PReTTy2 } \\
\text { (Progressive } \\
\text { Resistance Training } \\
\text { in Type 2Diabetes) } \\
\text { Trial }\end{array}$ & $\begin{array}{l}2019 \\
\text { China }\end{array}$ & $\begin{array}{l}265 \\
\text { patients }\end{array}$ & $\begin{array}{l}\text { Progressive } \\
\text { Resistance } \\
\text { exercise }\end{array}$ & $\begin{array}{l}12 \text { weeks } \\
\text { The participants } \\
\text { performed one } \\
\text { training session } \\
\text { per week. } \\
\text { Participants } \\
\text { accomplished } \\
\text { every session } \\
\text { within } \\
\text { 5-10 min weekly } \\
\text { during the study } \\
\text { and were } \\
\text { supervised by } \\
\text { qualified trainers. }\end{array}$ & $\begin{array}{l}\text { At the end of the } \\
\text { study period there } \\
\text { was no statistically } \\
\text { significant difference } \\
\text { in the HbA1c } \\
\text { between all patients } \\
\text { inthe control and } \\
\text { PRT groups. All } \\
\text { patients in the control } \\
\text { groupat the } 3-\text { month } \\
\text { interval for HbA1c } \\
{[6.73 \pm 0.94(50} \\
\text { mmol } / \mathrm{mol} \\
\pm 10.3 \mathrm{mmol} / \mathrm{mol})] \\
\text { vs. all patients in the } \\
\text { PRT group } 6.63 \\
\pm 0.98(49 \mathrm{mmol} / \mathrm{mol} \\
\pm 10.7 \mathrm{mmol} / \mathrm{mol}) \mathrm{p} \\
=0.427] \mathrm{P}=0.554\end{array}$ & $\begin{array}{l}\text { (Hangping } \\
\text { \& Xiaona, } \\
\text { 2019) }\end{array}$ \\
\hline 10. & $\begin{array}{l}\text { Effects of Three Type } \\
\text { Exercise Training } \\
\text { Programs onFBS and } \\
\text { HbA1C of Elderly } \\
\text { Men with Type } 2 \\
\text { Diabetes }\end{array}$ & $\begin{array}{l}2017 \\
\text { Iran }\end{array}$ & $52 \mathrm{~T} 2 \mathrm{DM}$ & $\begin{array}{l}\text { Training } \\
\text { Combine } \\
\text { Aerobik and } \\
\text { Resistance } \\
\text { Exercise }\end{array}$ & $\begin{array}{l}10 \text { weeks } \\
\text { Three times per } \\
\text { week for the } \\
\text { ATG and } \\
\text { RTG groups and } \\
\text { intermittent a } \\
\text { sessions for the } \\
\text { SAG group, } \\
\text { Each session } \\
\text { lasted } \\
\text { approximately } 90 \\
\text { minutes. }\end{array}$ & $\begin{array}{l}\text { The mean ( } \pm \text { standard } \\
\text { deviation) age of } \\
\text { participantswas } \\
53.24( \pm 1.05) \text { years } \\
\text { old. FBS decreased } \\
\text { significantly in all } \\
\text { studied groups (P- } \\
\text { value<0.05); and } \\
\text { HbA1c decreased } \\
\text { significantly in RTG } \\
\text { and SAG groups } \\
\text { (P-value<0.05), but, } \\
\text { there were no } \\
\text { significant }\end{array}$ & $\begin{array}{l}\text { (Siavoshy } \\
\& \\
\text { Heidarianp } \\
\text { our, 2017) }\end{array}$ \\
\hline
\end{tabular}


STRADA Jurnal Ilmiah Kesehatan

DOI: $10.30994 /$ sjik.v9i2.318

ISSN: 2252-3847 (print); 2614-350X (online)

Vol.9 No. 2 November 2020 Page.456-470

\begin{tabular}{|c|c|c|c|c|c|c|c|}
\hline No & Title & $\begin{array}{l}\text { Year, } \\
\text { Contry }\end{array}$ & $\begin{array}{l}\text { Sample } \\
\text { Size }\end{array}$ & $\begin{array}{l}\text { Type of } \\
\text { Exercise }\end{array}$ & $\begin{array}{c}\text { Intensity of } \\
\text { Exercise }\end{array}$ & $\begin{array}{c}\text { Finding/ } \\
\text { conclusion }\end{array}$ & Reference \\
\hline & & & & & & $\begin{array}{l}\text { differences in } \mathrm{HbA} 1 \mathrm{c} \\
\text { between groups }\end{array}$ & \\
\hline 11. & $\begin{array}{l}\text { Effect of regular } \\
\text { exercise training on } \\
\text { changes in } \mathrm{HbA} \mathrm{c} \text {, } \\
\mathrm{BMI} \text { and } \mathrm{VO}_{2} \text { max } \\
\text { among patients with } \\
\text { type } 2 \text { diabetes } \\
\text { mellitus: an } 8 \text {-year } \\
\text { trial }\end{array}$ & $\begin{array}{l}2017 \\
\text { Iran }\end{array}$ & $\begin{array}{l}65 \\
\text { patients } \\
\text { with } \\
\text { T2DM }\end{array}$ & $\begin{array}{l}\text { Regular } \\
\text { exercise } \\
\text { training }\end{array}$ & $\begin{array}{l}52 \text { weeks : three } \\
\text { sessions per } \\
\text { week (with } \\
\text { duration of } 15-40 \\
\text { min). }\end{array}$ & $\begin{array}{l}\text { Our long-term } \\
\text { exercise training } \\
\text { program had a } \\
\text { significant effect on } \\
\mathrm{HbA} 1 \mathrm{C}, \mathrm{BMI} \text { and } \\
\mathrm{VO}_{2} \text { max }(\mathrm{P}<0.05) \text {. } \\
\text { Compared with } \\
\text { patients in the control } \\
\text { group, HbA1c was } \\
\text { significantly reduced } \\
\text { and BMI and } \mathrm{VO}_{2} \\
\text { max were } \\
\text { significantly } \\
\text { improved among the } \\
\text { experiment group }\end{array}$ & $\begin{array}{l}\text { (Najafipou } \\
\text { r \& } \\
\text { Mobasseri, } \\
\text { 2017) }\end{array}$ \\
\hline 12. & $\begin{array}{l}\text { The Effect of } 12 \\
\text { Weeks Aerobic, } \\
\text { Resistance, and } \\
\text { Combined Exercises } \\
\text { on Omentin-1 Levels } \\
\text { and Insulin } \\
\text { Resistance among } \\
\text { Type } 2 \text { Diabetic } \\
\text { Middle-Aged Women }\end{array}$ & $\begin{array}{l}2017 \\
\text { Canada }\end{array}$ & $\begin{array}{l}52 \\
\text { participant } \\
\text { s (control } \\
\text { group 15, } \\
\text { AE group } \\
12, \mathrm{RE} \\
\text { group 12, } \\
\text { and CE } \\
\text { group 13) }\end{array}$ & $\begin{array}{l}\text { Aerobic } \\
\text { exercise } \\
\text { Resistance } \\
\text { exercise } \\
\text { Combine } \\
\text { Aerobic and } \\
\text { Resistance } \\
\text { exercise }\end{array}$ & $\begin{array}{l}10 \text { weeks } \\
\text { AE group : } 20 \\
\text { minutes streching } \\
\& \text { jogging } \\
25 \text { minutes main } \\
\text { exercise. } \\
\text { RE group: three } \\
\text { session per weeks } \\
\text { consist three sets } \\
\text { and eight } \\
\text { repetitions per } \\
\text { sets }\end{array}$ & $\begin{array}{l}\text { Glucose, } \mathrm{mg} / \mathrm{dL} \\
\text { Control group } \\
\text { (P value 0.06) } \\
\text { RE group } \\
\text { (P value } 0.005) \\
\text { AE group } \\
\text { (P value } 0.001) \\
\text { Combination } \\
\text { (P value } 0.001) \\
\text { AE group and } \\
\text { Combination (AE and } \\
\text { RE) was significantly } \\
\text { reduced glucose. }\end{array}$ & $\begin{array}{l}\text { (AminiLari } \\
\& \\
\text { Fararouei, } \\
\text { 2017) }\end{array}$ \\
\hline 13. & $\begin{array}{l}\text { Effects of Exercise in } \\
\text { the Fasted and } \\
\text { Postprandial State on } \\
\text { Interstitial Glucose } \\
\text { in Hyperglycemic } \\
\text { Individuals }\end{array}$ & $\begin{array}{l}2017 \\
\text { Norway }\end{array}$ & $\begin{array}{l}12 \\
\text { participant }\end{array}$ & $\begin{array}{l}\text { Aerobic } \\
\text { exercise }\end{array}$ & $\begin{array}{l}5 \text { weeks. } \\
\text { The exercise } \\
\text { sessions in both } \\
\text { ExBr and BrEx } \\
\text { consisted of } 60 \\
\text { min of treadmill } \\
\text { walking } \\
\text { at an individually } \\
\text { standardized } \\
\text { speed at } 8 \% \\
\text { inclination. }\end{array}$ & $\begin{array}{l}\text { Postprandial exercise } \\
\text { also decreased the } \\
\text { glycemic variability } \\
\text { compared to the } \\
\text { control day } \\
(1.22 \pm 0.49 \text { vs. } 1.58 \\
\pm 0.52 \mathrm{mmol} \cdot \mathrm{L}-1, \mathrm{p}= \\
0.015)\end{array}$ & $\begin{array}{l}\text { (Nygaard } \\
\& \\
\text { Rønnestad, } \\
\text { 2017) }\end{array}$ \\
\hline 14. & $\begin{array}{l}\text { Resistance Training } \\
\text { Improves Muscle } \\
\text { Function } \\
\text { and Cardiometabolic } \\
\text { Risks But Not } \\
\text { Quality } \\
\text { of Life in Older } \\
\text { People With Type } 2 \\
\text { Diabetes } \\
\text { Mellitus: A }\end{array}$ & $\begin{array}{l}2016 \\
\text { Taiwan }\end{array}$ & $\begin{array}{l}30 \text { people } \\
\text { with } \\
\text { T2DM }\end{array}$ & $\begin{array}{l}\text { Resistance } \\
\text { Training }\end{array}$ & $\begin{array}{l}\text { Eight RT } \\
\text { exercises - the } \\
\text { chest press, } \\
\text { shoulder } \\
\text { press, biceps curl, } \\
\text { hip abduction, } \\
\text { standing hip } \\
\text { flexion, leg } \\
\text { press, standing } \\
\text { calf raise, and }\end{array}$ & $\begin{array}{l}\text { No significant } \\
\text { correlation was noted } \\
\text { between the half- } \\
\text { recovery time for } \\
\text { deoxyhemoglobin } \\
\text { levels after peak } \\
\text { exercise and HbA1c } \\
\text { levels at week } 0 \text { or } 12 \\
(\mathrm{P}>.05) \text {. }\end{array}$ & $\begin{array}{l}\text { (Hsieh, } \\
\text { Tseng, \& } \\
\text { Tseng, } \\
\text { 2018) }\end{array}$ \\
\hline
\end{tabular}


STRADA Jurnal Ilmiah Kesehatan

DOI: $10.30994 /$ sjik.v9i2.318

ISSN: 2252-3847 (print); 2614-350X (online)

Vol.9 No. 2 November 2020 Page.456-470

\begin{tabular}{|c|c|c|c|c|c|c|c|}
\hline No & Title & $\begin{array}{l}\text { Year, } \\
\text { Contry }\end{array}$ & $\begin{array}{c}\text { Sample } \\
\text { Size } \\
\end{array}$ & $\begin{array}{l}\text { Type of } \\
\text { Exercise }\end{array}$ & $\begin{array}{c}\text { Intensity of } \\
\text { Exercise } \\
\end{array}$ & $\begin{array}{c}\text { Finding/ } \\
\text { conclusion }\end{array}$ & Reference \\
\hline & $\begin{array}{l}\text { Randomized } \\
\text { Controlled Trial }\end{array}$ & & & & $\begin{array}{l}\text { abdominal } \\
\text { crunch-were } \\
\text { performed in } 3 \\
\text { sets of } 8 \text { to } 12 \\
\text { repetitions. } \\
\text { Between each } \\
\text { set, the } \\
\text { participants } \\
\text { rested for } 60 \text { to } \\
90 \text { seconds. RT } \\
\text { was performed } 3 \\
\text { times per week } \\
\text { for } 12 \text { weeks. }\end{array}$ & & \\
\hline 15. & $\begin{array}{l}\text { Effects of } 12 \text { weeks } \\
\text { combined aerobic and } \\
\text { resistance exercise on } \\
\text { heart rate variability } \\
\text { in type } 2 \text { diabetes } \\
\text { mellitus patients }\end{array}$ & $\begin{array}{l}2016 \\
\text { Korea }\end{array}$ & $\begin{array}{l}16 \\
\text { patients } \\
\text { T2DM }\end{array}$ & $\begin{array}{l}\text { Combined } \\
\text { aerobic and } \\
\text { resistance } \\
\text { exercise }\end{array}$ & $\begin{array}{l}8 \text { weeks } \\
\text { Stretching as a } \\
\text { warm-up and } \\
\text { cool-down was } \\
\text { performed } \\
\text { for } 10 \text { minutes } \\
\text { until } 30 \text { minutes. } \\
\text { The aerobic } \\
\text { exercise consisted } \\
\text { of } 2 \text { sets of } 9 \\
\text { exercise items } \\
\text { using weight } \\
\text { machines with } 8- \\
12 \text { repetitions for } \\
30 \text { minutes at an } \\
\text { I-RM of } 60-80 \% \text {. }\end{array}$ & $\begin{array}{l}\text { Significant } \\
\text { correlation was noted } \\
\text { in Blood glucose } \\
(p<0.001) \text { and } \mathrm{HbA} 1 \mathrm{c} \\
(\mathrm{p}<0.001) .\end{array}$ & $\begin{array}{l}\text { (Kang \& } \\
\text { Ko, 2016) }\end{array}$ \\
\hline 16. & $\begin{array}{l}\text { Finding the Optimal } \\
\text { volume and intensity } \\
\text { of Resistance } \\
\text { Training Exercise for } \\
\text { Type } 2 \text { Diabetes: The } \\
\text { FORTE Study, a } \\
\text { Randomized Trial }\end{array}$ & $\begin{array}{l}2017 \\
\text { Canada }\end{array}$ & $\begin{array}{l}62 \\
\text { participant }\end{array}$ & $\begin{array}{l}\text { Resistance } \\
\text { exercise } \\
\text { from low } \\
\text { intensity } \\
\text { until higher } \\
\text { intensity. }\end{array}$ & $\begin{array}{l}24 \text { weeks } \\
\text { RT1 (Usual Care) } \\
\text { Intensity : Low } \\
\text { intensity (15-RM) } \\
\text { Repetitions : } \\
\text { High (15) } \\
\text { Sets : } 2 \\
\text { RT2 } \\
\text { Intensity : High } \\
\text { intensity (7-RM) } \\
\text { Repetitions : Low } \\
\text { (7) } \\
\text { Sets : } 3 \\
\text { RT3 } \\
\text { Intensity : Low } \\
\text { intensity } \\
\text { Repetitions : } \\
\text { High (15) } \\
\text { Sets : 2 }\end{array}$ & $\begin{array}{l}\text { Fasting glucose was } \\
\text { Significantly } \\
\text { different between } \\
\text { groups (RT1 differed } \\
\text { significantly } \\
\text { from RT3; RT1-RT3 } \\
=1.07 ; 95 \% \text { CI } \\
\left(\begin{array}{l}2.13, \quad 0.2) ; p< \\
0.05) .\end{array}\right.\end{array}$ & $\begin{array}{l}\text { (Yang \& } \\
\text { Swardfage } \\
\text { r, 2017) }\end{array}$ \\
\hline 17. & $\begin{array}{l}\text { Effect of 12-Month } \\
\text { Resistance Training } \\
\text { on Changes in } \\
\text { Abdominal } \\
\text { Adipose Tissue and }\end{array}$ & $\begin{array}{l}2019 \\
\text { China }\end{array}$ & $\begin{array}{l}93 \\
\text { participant }\end{array}$ & $\begin{array}{l}\text { Resistance } \\
\text { Training } \\
\text { and } \\
\text { Aerobic } \\
\text { Training }\end{array}$ & $\begin{array}{l}28 \text { month } \\
\text { resistance } \\
\text { training } 3 \\
\text { days/week with a } \\
\text { bungee cord }\end{array}$ & $\begin{array}{l}\text { No significant } \\
\text { differences were } \\
\text { noted in RT } \\
\text { intervention the } \\
\text { following parameters: }\end{array}$ & $\begin{array}{l}\text { Yan \& } \\
\text { Dai, 2019) }\end{array}$ \\
\hline
\end{tabular}




\section{STRADA Jurnal Ilmiah Kesehatan}

DOI: $10.30994 /$ sjik.v9i2.318

ISSN: 2252-3847 (print); 2614-350X (online)

Vol.9 No.2 November 2020 Page.456-470

\begin{tabular}{|c|c|c|c|c|c|c|c|}
\hline No & Title & $\begin{array}{l}\text { Year, } \\
\text { Contry }\end{array}$ & $\begin{array}{l}\text { Sample } \\
\text { Size }\end{array}$ & $\begin{array}{l}\text { Type of } \\
\text { Exercise }\end{array}$ & $\begin{array}{l}\text { Intensity of } \\
\text { Exercise }\end{array}$ & $\begin{array}{c}\text { Finding/ } \\
\text { conclusion }\end{array}$ & Reference \\
\hline & $\begin{array}{l}\text { Metabolic Variables } \\
\text { in Patients with } \\
\text { Prediabetes: A } \\
\text { Randomized } \\
\text { Controlled Trial }\end{array}$ & & & & $\begin{array}{l}\text { The resistance } \\
\text { training } \\
\text { sessions took } \\
\text { approximately } 50 \\
\text { minutes to } \\
\text { complete } \\
\text { The aerobic } \\
\text { training program } \\
\text { required } \\
\text { participants to } \\
\text { exercise } 3 \\
\text { days/week for } 60 \\
\text { minutes/session } \\
\text { (including 5-10 } \\
\text { minutes of warm- } \\
\text { up and 5-10 } \\
\text { minutes } \\
\text { offlexibility } \\
\text { exercises). }\end{array}$ & $\begin{array}{l}\text { Hba1c (group-by- } \\
\text { time interaction } \\
\mathrm{P}=0.573 \text { ) } \\
\text { In withingroup } \\
\text { analyses, decreases in } \\
\text { HbA1c were only } \\
\text { observed in } \\
\text { AT (group-by-time } \\
\text { interaction } \mathrm{P}=0.033 \text { ) }\end{array}$ & \\
\hline 18. & $\begin{array}{l}\text { Effects of combined } \\
\text { aerobic and resistance } \\
\text { training } \\
\text { on the glycolipid } \\
\text { metabolism and } \\
\text { inflammation } \\
\text { levels in type } 2 \\
\text { diabetes mellitus }\end{array}$ & $\begin{array}{l}2015 \\
\text { China }\end{array}$ & $\begin{array}{l}42 \\
\text { diabetes } \\
\text { patients }\end{array}$ & $\begin{array}{l}\text { Combined } \\
\text { aerobic and } \\
\text { resistance } \\
\text { training }\end{array}$ & $\begin{array}{l}12 \text { weeks } \\
\text { Aerobic exercise } \\
\text { was conducted } \\
\text { for a total of } 40- \\
60 \text { minutes per } \\
\text { session, } 3 \\
\text { sessions per } \\
\text { week. }\end{array}$ & $\begin{array}{l}\text { In the CT group, } \\
\text { FBG, PBG, PIN, and } \\
\text { HbA1c decreased } \\
\text { significantly after } \\
\text { treatment }(\mathrm{p}<0.05, \\
<0.01)\end{array}$ & $\begin{array}{l}\text { (Yuan Liu, } \\
\text { Liu, \& Cai, } \\
\text { 2015) }\end{array}$ \\
\hline
\end{tabular}

\section{DISCUSSION}

Regular physical exercise is highly recommended in the management of type 2 diabetes mellitus (T2DM). Physical activity includes all movement that increases energy use, whereas exercise is planned and structured physical activity. The previous study pointed that there were 3 types of physical exercise; aerobic exercise, resistance exercise and flexibility and balance exercise. These can be done by the T2DM patients as an intervention for glycemic control (Colberg \& Sigal, 2016).Exercise improves blood glucose controlin type 2 diabetes, reduces cardiovascular risk factors, contributes to weight loss, and improves well-being (Xie, Li, Wang, Li, \& Chen, 2015) (Lin \& Zhang, 2015). It can decrease the levels of inflammatory factors such as TNF- $\alpha$, IL-6, CRP and leptin while increasing the levels of anti-inflammatory factors such as IL-4, IL-10, and adiponectin in diabetes patients. Exercise also reduces the inflammation level and improves IR by enhancing anti-stress and anti-oxidative effects (Kurniawati \& Kusumawati, 2020). Exercise therapy is an effective method for treating diabetes mellitus. Many studies show aerobic exercise including home-based exercise decreases blood glucose, lipid, and HbAlc levels as well as improves insulin sensitivity, blood pressure, in T2DM patient (Ghalavand \& Rooholah, 2016) (Gholami \& Nikookheslat, 2018) (Nygaard \& Rønnestad, 2017).

This systematic review, the authors reviewed 18 research articles about various physical exercise (aerobics, resistance exercie, and a combination of aerobics and resistance exercise) related to glycemic control (blood sugar and Hba1c). 


\section{STRADA Jurnal Ilmiah Kesehatan}

DOI: $10.30994 /$ sjik.v9i2.318

ISSN: 2252-3847 (print); 2614-350X (online)

Vol.9 No.2 November 2020 Page.456-470

\section{Aerobic Exercise}

The American College of Sports Medicine (ACSM) defines aerobic exercise as any activity that uses large muscle groups, can be maintained continuously and is rhythmic in nature (Chodzko-Zajko \& Proctor, 2019).As the name implies, muscle groups activated by this type of exercise rely on aerobic metabolism to extract energy in the form of adenosine triphosphate (ATP) from amino acids, carbohydrates and fatty acids. In this systematic review, the study's result show that one intervention aerobic exercise has a significant for reducing HbA1c and blood glucose according to study from (Ghalavand \& Rooholah, 2016) that 8 weeks with duration 10 -30 minutes duration per weeks make significant decrease in fasting blood glucose $(\mathrm{P}=0.017)$ and $\mathrm{HbA1C}(\mathrm{P}=0.045)$. Then, study from (Gholami \& Nikookheslat, 2018) 12 weeks aerobic exercise(AE), fasting glucose levels in the exercise group significantly decreased from $11.22 \pm 3.8 \mathrm{mmol} / \mathrm{L}$ to $8.58 \pm 2.7 \mathrm{mmol} / \mathrm{L}$. $2 \mathrm{hpp}$ glucose level also decreased significantly from $15.76 \pm 2.5 \mathrm{mmol} / \mathrm{L}$ to $14.21 \pm 2.5$ $\mathrm{mmol} / \mathrm{L}$ indicating $9 \%$ reduction in the mean value $(\mathrm{P}=0.009)$. In the exercise group, HbA1c significantly decreased from $8.3 \% \pm 1.4 \%$ at week to $7.7 \% \pm 1.5 \%$ at week.

Thrid study from (Nygaard \& Rønnestad, 2017) 5 weeks aerobic exercise postprandial exercise decreased the mean of the 10 highest glucose values measured in each individual $(8.6 \pm 1.9 \mathrm{mmol} \cdot \mathrm{L}-1)$ over 22 hours compared to both the control day $(9.3$ \pm 2.1 mmol.L-1). From all the study, average duration 8 weeks and 20-40 minutes intensity of aerobic exercise can decrease level of $\mathrm{HbA} 1 \mathrm{c}$ and blood sugar. During aerobic exercise, muscle contraction stimulates an increase in AMPK activity (AMP-activated protein kinase). This activation then stimulates GLUT4 translocation into the cell membrane, thereby increasing the glucose uptake (Bird \& Hawley, 2017). In T2DM, there are deficiencies in the insulin receptors which results in impaired glucose uptake and GLUT4 translocation. However, exercise therapy could restore the defects of insulin by providing GLTU4 translocation (Bird \& Hawley, 2017).

\section{Resistance Exercise}

Resistance training benefits for individuals with type 2 diabetes include improvements in glycemic control, insulinresistance, fat mass, blood pressure, strength, and lean body mass (Colberg, 2016). Resistance training-induced physiological stimuli and/or specific molecular signaling cascades can facilitate a number of physiological adaptations in individuals, and mitigate the diabetes complications (Shaw, Sicree, \& Zimmet, 2010). For instance, RE induces beneficial changes in insulin sensitivity through increased

skeletal muscle mass, glucose storage, enhanced glucose clearance from circulation, and improved

mitochondrial oxidative capacity (Pesta \& Goncalves, 2017). Improved insulin sensitivity in T2D was associated with RE-induced ( $67 \%$ 1RM) loss of abdominal fat and increased muscle density(Yubo Liu \& Ye, 2019).

Our study review that decreased blood glucose and HbA1c with resistance exercise (RE) as single intervention have significant and no significant result. First, study from (Buch et al., 2019) 10 weeks Circuit Resistance Training (CRT) 3 home sessions per week has significant dercrease glycemic control Fasting Plasma Glucose (FPG) and HbA1c $(\mathrm{p}=0.006)$. Second, study from (Toghi-Eshghi \& Reza, 2019) during 10 weeks performed 40 minutes $\mathrm{RE}$ and te result is Blood glucose decrease in treatment group exercise from 8.262.5 to $7.462 .6 \mathrm{mmol} / \mathrm{L}$. Third study, from (Yang \& Swardfager, 2017) 


\section{STRADA Jurnal Ilmiah Kesehatan}

DOI: $10.30994 /$ sjik.v9i2.318

ISSN: 2252-3847 (print); 2614-350X (online)

Vol.9 No.2 November 2020 Page.456-470

in 62 participant during 24 weeks with RE from low intensity until higher intensity has result for fasting glucose was significantly different between groups (RT1 different significantly from RT3; RT1-RT3 $=1.07$; $95 \%$ CI $(2.13,0.2)$; $\mathrm{p}<0.05)$. Similar to aerobic exercise, resistance exercises are useful therapeutic tools in the management of T2DM. In addition, it was also proven to be safe and efficacious for the elderly insulin resistant diabetic patients. Resistance exercise has been reported to enhance insulin sensitivity, daily energy expenditure and quality of life(Bird \& Hawley, 2017). Furthermore, resistance training has the potential to increase muscle strength, lean muscle mass and bone mineral density, which could enhance both functional status and glycemic control (Patel \& Alkhawam, 2017).

Nevertheless, many study showed significant result in the other hand there are no statistically significant for decrease $\mathrm{HbA} 1 \mathrm{c}$ level in T2DM patients with RE intervention in study from(Chen et al., 2020) (Hangping \& Xiaona, 2019) (Hsieh et al., 2018) between intervention group and control group.

\section{Kombinasi AE dan RE}

In the present study, T2DM patients performed combination of aerobic exercises and resistance training for 10 to 12 weeks. The study's result before was related to the results of the current study in combination of aerobic and resistance exercise were the most common types of physical exercise used for maintaining glycemic control respectively. Compared with supervised aerobic or supervised resistance exercise alone, combined exercise showed there to be more of an improvement in HbA1c levels and blood sugar.

First study from study (Siavoshy \& Heidarianpour, 2017) 52 T2DM patient in Iran during 10 weeks combine (AE and RE) the result is FBS decreased significantly in all studied groups $(\mathrm{P}-\mathrm{value}<0.05)$ and HbA1c decreased significantly in RTG and SAG groups (P-value $<0.05$ ). Second study from (AminiLari \& Fararouei, 2017) 52 participants in Canada doing combine $\mathrm{AE}$ and $\mathrm{RE}$ at 8 weeks and the result is $\mathrm{AE}$ group and Combination (AE and RE) was significantly reduced glucose with (P value 0.001). Third study from (Kang \& Ko, 2016) in South Korea with 16 participant doing combine Ae and RE during 10 weeks and the result is significant correlation was noted in Blood glucose $(\mathrm{p}<0.001)$ and HbA1c $(\mathrm{p}<0.001)$. Fourth study from (Yuan Liu, 2015) in China with 42 T2DM pastient doing combine AE and RE during 12 weeks and the result is FBG, PBG and HbA1c decreased significantly after treatment $(\mathrm{p}<0.05,<0.01)$. The limitation of this study was the variation of the intervention's duration in many studies . Two studies had an intervention duration that was less than standart 8 days. The other studies were done over more than 12 week.

\section{CONCLUSION}

The present systematic review showed the effectiveness of various physical exercise on glycemic control (HbA1c and blood glucose T2DM patinet. From the published data, it can be concluded that exercise-based research on a schedule of minimum 2 or 3 times a week for 30 to 60 minutes per session over 2 months was widely used and significant at reducing blood glucose and hemoglobin A1c (HbA1c). This systematic review could be used as evidence when carrying out combined aerobic and resistance exercise as good choice physical exercise interventions for the purpose of glycemic control on patient with diabetes type 2 


\section{STRADA Jurnal Ilmiah Kesehatan}

DOI: $10.30994 /$ sjik.v9i2.318

ISSN: 2252-3847 (print); 2614-350X (online)

Vol.9 No.2 November 2020 Page.456-470

\section{REFERENCES}

AminiLari, Z., \& Fararouei, M. (2017). The effect of 12 weeks aerobic, resistance, and combined exercises on omentin-1 levels and insulin resistance among type 2 diabetic middle-aged women. Diabetes and Metabolism Journal, 41(3), 205-212. https://doi.org/10.4093/dmj.2017.41.3.205

Banitalebi, E., Kazemi, A. R., \& Faramarzi, M. (2019). Effects of sprint interval or combined aerobic and resistance training on myokines in overweight women with type 2 diabetes: A randomized controlled trial. Life Sciences, 217, 101-109. https://doi.org/10.1016/j.lfs.2018.11.062

Bird, S. R., \& Hawley, J. A. (2017). Update on the effects of physical activity on insulin sensitivity in humans. BMJ Open Sport and Exercise Medicine, 2(1), 1-26. https://doi.org/10.1136/bmjsem-2016-000143

Brinkmann, C., Weh-Gray, O., \& Brixius, K. (2019). Effects of exercising before breakfast on the health of T2DM patients-A randomized controlled trial. Scandinavian Journal of Medicine and Science in Sports, 29(12), 1930-1936. https://doi.org/10.1111/sms.13543

Buch, A., Eldor, R., \& Kis, O. (2019). The effect of circuit resistance training, empagliflozin or "vegeterranean diet" on physical and metabolic function in older subjects with type 2 diabetes: A study protocol for a randomized control trial (CEV65 trial). BMC Geriatrics, 19(1), 1-12. https://doi.org/10.1186/s12877-019-1219-7

Chen, S. M., Shen, F. C., \& Chen, J. F. (2020). Effects of resistance exercise on glycated hemoglobin and functional performance in older patients with comorbid diabetes mellitus and knee osteoarthritis: A randomized trial. International Journal of Environmental Research and Public Health, 17(1), 1-13. https://doi.org/10.3390/ijerph17010224

Chodzko-Zajko, W. J., \& Proctor, D. N. (2019). Exercise and physical activity for older adults. Medicine and Science in Sports and Exercise, 41(7), 1510-1530. https://doi.org/10.1249/MSS.0b013e3181a0c95c

Colberg, S. R., \& Sigal, R. J. (2016). Physical activity/exercise and diabetes: A position statement of the American Diabetes Association. Diabetes Care, 39(11), 2065-2079. https://doi.org/10.2337/dc16-1728

Dixit, S., \& Maiya, A. (2017). Effect of moderate-intensity aerobic exercise on glycosylated haemoglobin among elderly patients with type 2 diabetes \& peripheral neuropathy. IJMR. https://doi.org/DOI: 10.4103/ijmr.IJMR_699_14

Ghalavand, A., \& Rooholah, R. (2016). Effects of aerobic training on FBS , HbA1C , Fructosamine and plasma lipid profile in male with type 2 diabetes. International Journal of Medical Research \& Health Sciences, 5(6), 27-33.

Gholami, F., \& Nikookheslat, S. (2018). Effect of aerobic training on nerve conduction in men with type 2 diabetes and peripheral neuropathy: A randomized controlled trial. Neurophysiologie Clinique, https://doi.org/10.1016/j.neucli.2018.03.001

Hangping, Z., \& Xiaona, Q. (2019). The impact on glycemic control through progressive resistance training with bioDensity TM in Chinese elderly patients with type 2 diabetes: The PReTTy2 (Progressive Resistance Training in Type 2 Diabetes) Trial. Diabetes Research and Clinical Practice, 150, 64-71. https://doi.org/10.1016/j.diabres.2019.02.011

Hsieh, P. L., Tseng, C. H., \& Tseng, Y. J. (2018). Resistance Training Improves Muscle 


\section{STRADA Jurnal Ilmiah Kesehatan}

DOI: $10.30994 /$ sjik.v9i2.318

ISSN: 2252-3847 (print); 2614-350X (online)

Vol.9 No.2 November 2020 Page.456-470

Function and Cardiometabolic Risks but Not Quality of Life in Older People with Type 2 Diabetes Mellitus: A Randomized Controlled Trial. Journal of Geriatric Physical Therapy, 41(2), 65-76. https://doi.org/10.1519/JPT.0000000000000107

IDF. (2017). International Diabetes Federation (IDF) Atlas Eighth edition 2017.

Kang, S. J., \& Ko, K. J. (2016). Effects of 12 weeks combined aerobic and resistance exercise on heart rate variability in type 2 diabetes mellitus patients. Journal of Physical Therapy Science, 28(7), 2088-2093. https://doi.org/10.1589/jpts.28.2088

Kurniawati, Y., \& Kusumawati, M. (2020). Effectiveness of Physical Exercise on the Glycemic Control of Type 2 Diabetes Mellitus Patients: A Systematic Review. Jurnal Ners, 14(3), 199. https://doi.org/10.20473/jn.v14i3.17059

Lin, X., \& Zhang, X. (2015). Effects of exercise training on cardiorespiratory fitness and biomarkers of cardiometabolic health: A systematic review and meta-analysis of randomized controlled trials. Journal of the American Heart Association, 4(7), 1-28. https://doi.org/10.1161/JAHA.115.002014

Liu, Yuan, Liu, S. xin, \& Cai, Y. (2015). Effects of combined aerobic and resistance training on the glycolipid metabolism and inflammation levels in type 2 diabetes mellitus. Journal of Physical Therapy Science, 27(7), 2365-2371. https://doi.org/10.1589/jpts.27.2365

Liu, Yubo, \& Ye, W. (2019). Resistance Exercise Intensity is Correlated with Attenuation of HbAlc and Insulin in Patients with Type 2 Diabetes: A Systematic Review and Meta-Analysis. International Journal of Env Res and Pub Health. https://doi.org/10.3390/ijerph16010140

Najafipour, F., \& Mobasseri, M. (2017). Effect of regular exercise training on changes in HbA1c, BMI and VO 2 max among patients with type 2 diabetes mellitus: An 8-year trial. BMJ Open Diabetes Research and Care, 5(1), 1-7. https://doi.org/10.1136/bmjdrc-2017-000414

Nygaard, H., \& Rønnestad, B. R. (2017). Effects of exercise in the fasted and postprandial state on interstitial glucose in hyperglycemic individuals. Journal of Sports Science and Medicine, 16(2), 254-263.

Patel, H., \& Alkhawam, H. (2017). Aerobic vs anaerobic exercise training effects on the cardiovascular system. World Journal of Cardiology, 9(2), 134. https://doi.org/10.4330/wjc.v9.i2.134

Pesta, D. H., \& Goncalves, R. L. S. (2017). Resistance training to improve type 2 diabetes : working toward a prescription for the future. Nutrition \& Metabolism (2017), 14, 1-10. https://doi.org/10.1186/s12986-017-0173-7

Shaw, J. E., Sicree, R. A., \& Zimmet, P. Z. (2010). Global estimates of the prevalence of diabetes for 2010 and 2030. Diabetes Research and Clinical Practice, 87(1), 4-14. https://doi.org/10.1016/j.diabres.2009.10.007

Siavoshy, H., \& Heidarianpour, A. (2017). Effects of Three Type Exercise Training Programs on FBS and HbA1C of Elderly Men with Type 2 Diabetes. Iranian Journal of Diabetes and Obesity, 9(1), 14-19.

Toghi-Eshghi, \& Reza, S. (2019). Morning (Fasting) vs Afternoon Resistance Exercise in Individuals with Type 1 Diabetes: A Randomized Crossover Study. Journal of Clinical Endocrinology and Metabolism, 104(11), 5217-5224. https://doi.org/10.1210/jc.2018-02384

Xie, C., Li, J., Wang, K., Li, Q., \& Chen, D. (2015). ScienceDirect Probiotics for the prevention of antibiotic- associated diarrhoea in older patients : A systematic review. 


\section{STRADA Jurnal Ilmiah Kesehatan}

DOI: $10.30994 /$ sjik.v9i2.318

ISSN: 2252-3847 (print); 2614-350X (online)

Vol.9 No.2 November 2020 Page.456-470

Travel Medicine and Infectious 1-7. https://doi.org/10.1016/j.tmaid.2015.03.001

Yan, J., \& Dai, X. (2019). Effect of 12-Month Resistance Training on Changes in Abdominal Adipose Tissue and Metabolic Variables in Patients with Prediabetes: A Randomized Controlled Trial. Journal of Diabetes Research, 2019, 1-11. https://doi.org/10.1155/2019/8469739

Yang, P., \& Swardfager, W. (2017). Finding the Optimal volume and intensity of Resistance Training Exercise for Type 2 Diabetes: The FORTE Study, a Randomized Trial. Diabetes Research and Clinical Practice, 130(416), 98-107. https://doi.org/10.1016/j.diabres.2017.05.019 